\title{
Cartilaginous Hamartoma
}

National Cancer Institute

\section{Source}

National Cancer Institute. Cartilaginous Hamartoma. NCI Thesaurus. Code C40424.

A hamartoma characterized by the presence of cartilaginous elements. 